\title{
Constraints on cosmological birefringence from PLANCK and Bicep2/Keck data
}

\author{
A. Gruppuso ${ }^{a, b}$, M. Gerbino ${ }^{c}$, P. Natoli ${ }^{d}$, L. Pagano $^{c}$, \\ N. MANDOLESI ${ }^{d, a}$ AND D. MolinaRI ${ }^{d}$ \\ ${ }^{a}$ INAF-IASF Bologna, \\ Istituto di Astrofisica Spaziale e Fisica Cosmica di Bologna, \\ Istituto Nazionale di Astrofisica, via Gobetti 101, I-40129 Bologna, Italy \\ ${ }^{b}$ INFN, Sezione di Bologna, Via Irnerio 46, I-40126 Bologna, Italy \\ ${ }^{c}$ Dipartimento di Fisica and INFN, Università di Roma La Sapienza, \\ P.le Aldo Moro 2, 00185, Rome, Italy \\ ${ }^{d}$ Dipartimento di Fisica e Scienze della Terra and INFN, Università degli Studi di \\ Ferrara, Via Saragat 1, I-44100 Ferrara, Italy
}

\begin{abstract}
The polarization of cosmic microwave background (CMB) can be used to constrain cosmological birefringence, the rotation of the linear polarization of $\mathrm{CMB}$ photons potentially induced by parity violating physics beyond the standard model. This effect produces non-null CMB cross correlations between temperature and B modepolarization, and between E- and B-mode polarization. Both crosscorrelations are otherwise null in the standard cosmological model. We use the recently released 2015 PLANCK likelihood in combination with the Bicep2/Keck/Planck (BKP) likelihood to constrain the birefringence angle $\alpha$. Our findings, that are compatible with no detection, read $\alpha=0.0^{\circ} \pm 1.3^{\circ}$ (stat) $\pm 1^{\circ}$ (sys) for PLANCK data and $\alpha=0.30^{\circ} \pm 0.27^{\circ}$ (stat) $\pm 1^{\circ}$ (sys) for BKP data.

We finally forecast the expected improvements over present constraints when the PLANCK BB, TB and EB spectra at high $\ell$ will be included in the analysis.
\end{abstract}

\section{Introduction}

It has become customary to use polarized cosmic microwave background $(\mathrm{CMB})$ data to constrain the cosmic birefringence effect [1, 2, 3], i.e. the in vacuo rotation of the photon polarization direction during propagation [4]. In general, such effect, which is naturally parameterized by an angle $\alpha$, results in a mixing between $\mathrm{Q}$ and $\mathrm{U}$ Stokes 
parameters that produces non-null $\mathrm{CMB}$ cross correlations between temperature and B mode-polarization, and between E- and B-mode polarization. Since these correlations are expected to be null under the parity conserving assumptions that is beneath the standard cosmological model, cosmic birefringence is a tracer of parity violating physics.

Several theoretical models exhibit cosmological birefringence. Any coupling of the electromagnetic (EM) field with axion-like particles [5] or a quintessence field [6] is expected to induce the effect, which can be also driven by quantum-gravity terms [7] or Chern-Simons type interactions [4] in the EM Lagrangian. Each particular model predicts a specific dependence of the CMB angular power spectra (henceforth APS) on $\alpha$, which can be complicated. In this paper, we restrict to the simplest case of constant $\alpha$, for which the effect can be parametrized as [8, 9 ]

$$
\begin{aligned}
C_{\ell}^{T E, o b s} & =C_{\ell}^{T E} \cos (2 \alpha), \\
C_{\ell}^{T B, o b s} & =C_{\ell}^{T E} \sin (2 \alpha), \\
C_{\ell}^{E E, o b s} & =C_{\ell}^{E E} \cos ^{2}(2 \alpha)+C_{\ell}^{B B} \sin ^{2}(2 \alpha), \\
C_{\ell}^{B B, o b s} & =C_{\ell}^{B B} \cos ^{2}(2 \alpha)+C_{\ell}^{E E} \sin ^{2}(2 \alpha), \\
C_{\ell}^{E B, o b s} & =\frac{1}{2}\left(C_{\ell}^{E E}-C_{\ell}^{B B}\right) \sin (4 \alpha),
\end{aligned}
$$

with $C_{\ell}^{X Y, o b s}$ and $C_{\ell}^{X Y}$ being the observed and the primordial (i.e. unrotated) APS for the $X Y$ spectrum $(X, Y=T, E$ or $B)$, i.e. the one that would arise in absence of birefringence. Note that we set the primordial $T B$ and $E B$ spectra to zero for the sake of simplicity, thereby assuming parity violation effect plays a negligible role up to $\mathrm{CMB}$ photon decoupling (this choice excludes e.g. chiral gravity theories).

The existing limits on $\alpha$, as obtained from CMB experiments 1 , 2, 3, 10, 11, 12, are shown in Fig. 1 (see first seven estimates). In short, almost all constraints are well compatible with a null effect, and the best available statistical errors are of order of $0.2^{\circ}$ with a systematic uncertainty estimated at the level of $0.5^{\circ}$. For comparison, still in Fig. 1, we anticipate the constraints we obtain from PLANCK and Bicep2/Keck/Planck (henceforth BKP) data in this paper (see the last two constraints). As discussed below the PLANCK constraints do not include $B$ information at $\ell>29$ while this is included in BKP combination.

This paper is organized as follows: in Section 2 we provide a description of the performed analysis; in Section 3 we report the datasets employed and the associated likelihood functions; in Section 4 we 


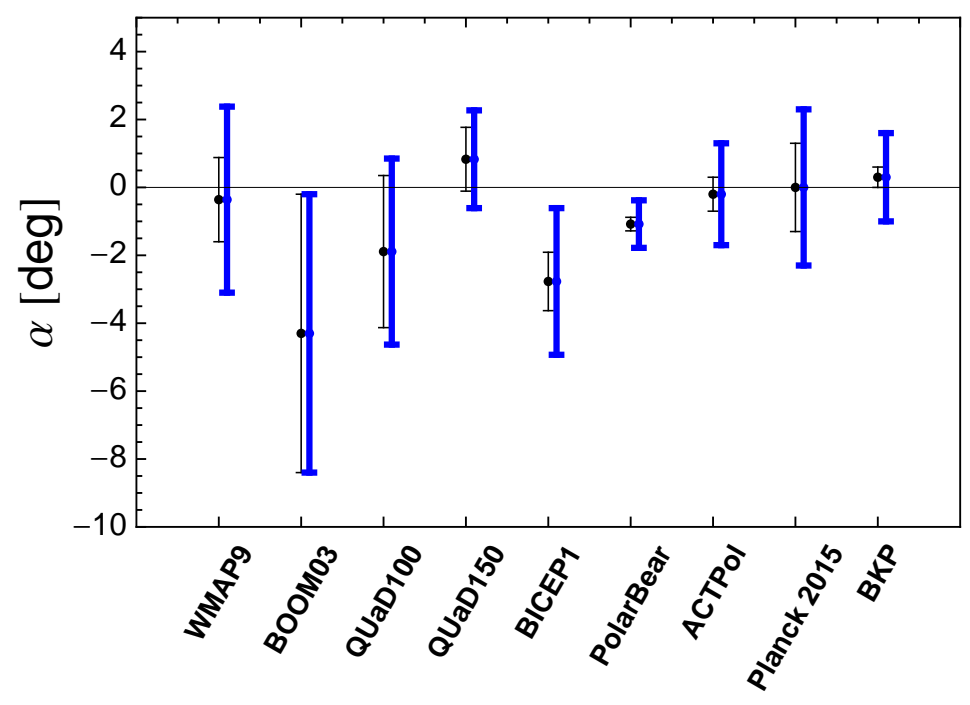

Figure 1: Constraints on $\alpha$ from several CMB experiments [1, 2, 3, 10, 11, 12]. Thin black error bars are for statistical uncertainties at $68 \%$ C.L.. Thick blue error bars are obtained by summing linearly the statistical and systematic uncertainties. The statistical uncertainty of BOOM03 do already contain a contribution from systematic effect. The last two constraints are derived in this paper. 
present the main constraints on $\alpha$ sampling on all standard $\Lambda \mathrm{CDM}$ parameters and as well on the tensor-to-scalar ratio, $r$. We also discuss the role of systematic uncertainties. We finally draw our conclusions in Section 5 providing also forecast for the statistical sensitivity that PLANCK data can reach when BB, TB and EB spectra will be included at high $\ell$ in the PLANCK likelihood.

\section{Description of the analysis}

CMB polarization arises at two distinct cosmological times: the recombination epoch $(z \simeq 1100)$ and the reionization era at $z \sim 10$ [13. When the CMB field is expanded in spherical harmonics, the first signal mostly shows up at high multipoles, since polarization is generated through a causal process and the Hubble horizon at last scattering only subtends a degree sized angle. The later reionization of the cosmic fluid at lower redshift impacts instead the lowest multipoles. These two regimes need to be properly treated when probing for cosmological birefringence. It is also interesting to separate the constraints on $\alpha$ between high and low $\ell$ as these can be ascribed to different epochs and, hence, physical conditions. Therefore, following [14], we perform a Monte Carlo Markov chain (MCMC) analysis making use of the publicly available code cosmomc [15, 16] to constrain $\alpha$ by considering two multipole ranges $11,2 \leq \ell \leq 29$ and $30 \leq \ell \leq 2500$. In practice, we first sample at low $\ell$ on $\alpha$, on the optical depth $\tau$ and on the scalar amplitude $A_{s}$, while fixing the remaining $\Lambda \mathrm{CDM}$ cosmological parameters to the PLANCK best-fit model derived from temperature and polarization (TT, TE, EE). We then focus on the high $\ell$, fixing $\tau$ to the best-fit value obtained in the former analysis and let the remaining parameters to vary. We call the above combination case $\mathrm{A}$. We report separately the constraints on $\alpha$ obtained by a single run on all multipoles. This is called case B in what follows and it is provided for comparison purposes. Moreover, we consider a third case, C, where we sample at the same time on two separate angles, namely $\alpha_{\text {low }}$ and $\alpha_{\text {high }}$, which rotate the APS independently in the two multipole regimes.

These three cases above are performed considering only scalar perturbations (i.e. $\Lambda \mathrm{CDM}+\alpha$ ) and also considering tensor perturbations (i.e. $\Lambda \mathrm{CDM}+\mathrm{r}+\alpha$ ). See Table 1 for a summary of all cases considered.

\footnotetext{
${ }^{1}$ The transition multipole between low and high $\ell$ is taken to be 29 following the low and high transition of the PLANCK likelihood (see also Section 3). The fine choice of this multipole does not impact significantly the results as long as it occurs in a region where the polarization signal is noise dominated [17].
} 
Table 1: Summary of the considered cases. See text.

\begin{tabular}{cll}
\hline case & description & color code \\
\hline A & $\begin{array}{l}\text { First run at low } \ell \text { on } \alpha, A_{s} \text { and } \tau \\
\text { (other parameters fixed to Planck best fit) }\end{array}$ & light blue \\
& $\begin{array}{l}\text { Second run at high } \ell \text { on } \Lambda \operatorname{CDM}(+\mathrm{r})+\alpha \\
\text { with } \tau \text { fixed to the first run best fit value }\end{array}$ & blue \\
B $\quad \begin{array}{l}\text { Single run on } \Lambda \mathrm{CDM}(+\mathrm{r})+\alpha \text { in all the } \ell \\
\text { range }\end{array}$ & red \\
$\mathrm{C} \quad$ & $\begin{array}{l}\text { Single run on } \Lambda \mathrm{CDM}(+\mathrm{r})+\alpha_{\text {low }}+\alpha_{\text {high }} \\
\text { where } \alpha_{\text {low }}\left(\text { dashed) and } \alpha_{\text {high }} \text { (solid) are }\right. \\
\text { defined in the low and high } \ell \text { range } \\
\end{array}$ & \\
& respectively & \\
\hline \hline
\end{tabular}

Gravitational lensing induces non zero TB and EB correlations through E to B mixing, an effect potentially observable at the sensitivity level of the PLANCK and BKP dataset. To disentangle it from cosmological birefringence, one should in principle model the latter within the CMB time evolution from last scattering to us. However, it can be shown 18, that for the model given in Eqs. (115), one can safely separate the birefringence and lensing contributions.

\section{Datasets and likelihoods}

We have used the PLANCK and BKP publicly available likelihood\$2 Here we list the several terms entering the likelihood functions [17, 19].

- Planck lowl. Planck low $\ell$ temperature likelihood. Based on the Planck 2015 low resolution CMB anisotropy map (Commander, see [20]).

- Planck lowTEB. Planck low $\ell$ temperature and polarization likelihood. Based on temperature (Commander) and polarization $(70 \mathrm{GHz})$ maps.

- Planck TT, TE, EE. PLANCK high $\ell$ temperature and polarization Likelihood. Based on TT, TE and EE cross-spectra.

- BKP BB. Bicep2/Keck likelihood with 353GHz PLANCK channel. Based on the first five band powers of the BB spectra [19].

${ }^{2}$ For PLANCK see http://pla.esac.esa.int/pla/ and for BKP see
http://bicepkeck.org/bkp_2015_release.html



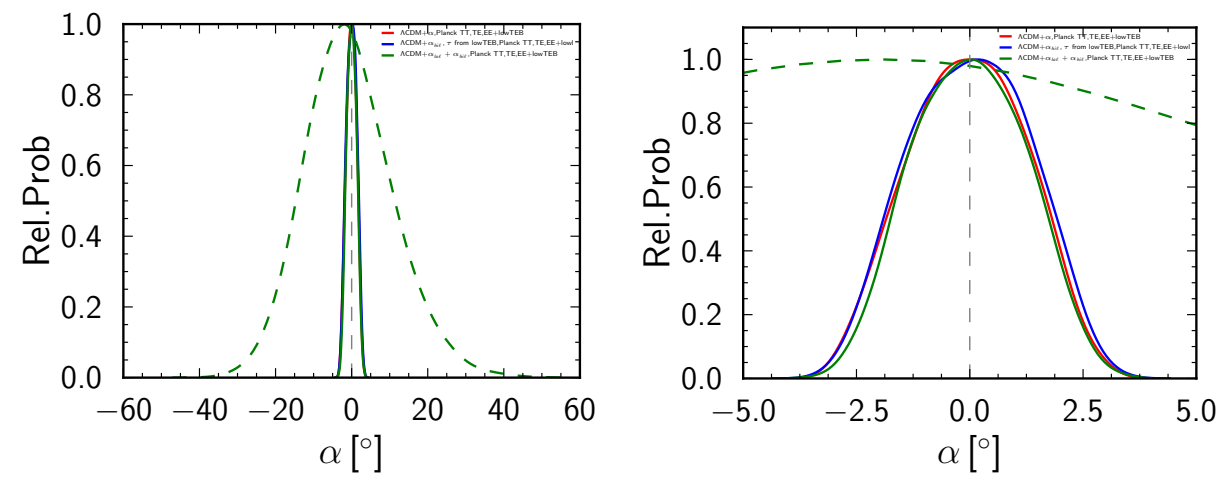

Figure 2: Left panel: Constraints on $\alpha$ from the three considered cases summarized in Table 1. Blue curves are for case A, red curves for case B and green curves for case $\mathrm{C}$ (solid green for $\ell \in[30,2500]$ and dashed green for $\ell \in[2,29])$. Right panel: zoom of the left panel.

- BKP EE, BB. Bicep2/Keck likelihood with 353GHz PlanCK channel. Based on the first five band powers of the BB, EE and EB spectra.

From Eqs. (1.5) it is clear that we are sensitive to the sign of $\alpha$ only when we use TB and EB information. This happens only when we employ Planck lowTEB and BKP EE, BB. Therefore, when the latter are not considered, in fact our constraints on $\alpha$ will be on its absolute value.

For each considered case, we employ the following likelihoods:

- Case A. First run Planck lowTEB. Second run Planck lowl + Planck TT, TE,EE.

- Case B. Single run with Planck lowTEB + Planck TT,TE,EE.

- Case C. Single run with Planck lowTEB + Planck TT, TE,EE.

All the above cases are analyzed for both $\Lambda \mathrm{CDM}+\alpha$ and $\Lambda \mathrm{CDM}+\mathrm{r}+\alpha$. Moreover, in the latter case we consider the likelihoods BKP BB or BKP $\mathrm{EE}, \mathrm{BB}$ either alone (with minimum multipole $\ell \simeq 50$ ) or in combination with PLANCK.

\section{Results}

\section{1 $\Lambda \mathrm{CDM}+\alpha$}

The $\Lambda \mathrm{CDM}+\alpha$ model is analyzed considering PLANCK data alone. 


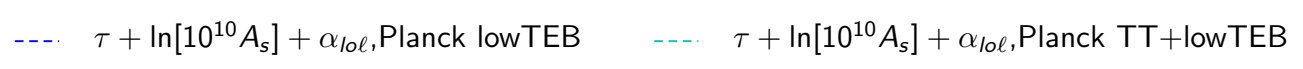

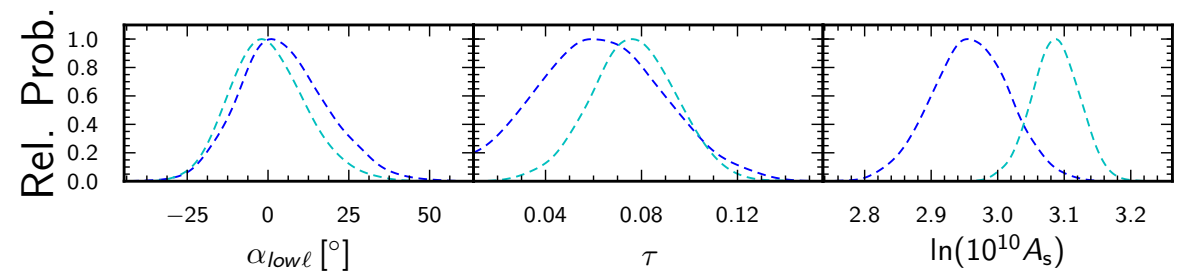

Figure 3: Posterior distributions of $\alpha, \tau$ and $A_{s}$ for the low $\ell$ run of case A. Blue curves consider temperature and polarization at low $\ell$ while light blue take into account polarization at low $\ell$ and temperature at all $\ell$.

The posterior distributions for $\alpha$ are shown in Fig. 2 and the corresponding constraints are reported in Table 2, The latter only account for statistical uncertainty (68\% C.L.). The companion "low- $\ell$ run" of case $\mathrm{A}$ is displayed in Fig. 3 . Fig. 4 shows that all the standard $\Lambda \mathrm{CDM}$ cosmological parameters are very stable against the inclusion of $\alpha$ in the MCMC and Fig. 5 shows how the same parameters are very stable in all three considered cases.

All the PLANCK constraints are compatible with zero even considering only the statistical uncertainty. Still without accounting for systematic uncertainty, the best constraint for PLANCK alone is $\alpha=$ $0.0^{\circ} \pm 1.3^{\circ}$. The standard error will improve significantly in a future version of the high $\ell$ likelihood which will employ the TB and EB spectra. Forecasts are provided below.

\section{2 $\Lambda \mathrm{CDM}+\mathrm{r}+\alpha$}

The $\Lambda \mathrm{CDM}+\mathrm{r}+\alpha$ model is analyzed with the following data set combinations: PLANCK alone, BKP and PLANCK plus BKP. Since all data in the BKP dataset come from the small $\left(f_{s k y} \simeq 1 \%\right)$ region observed by Bicep, we neglect the bias caused by counting twice the PLANCK dataset in this region, for the latter combination. As for the $\Lambda \mathrm{CDM}+\alpha$ model, all the estimates are reported in Table 2 .

- Planck data alone. The posterior distribution functions of $\alpha$ are displayed in Fig. 6. In Fig. 7 we show the low $\ell$ companion run of case A. The posterior distribution functions of the six standard cosmological parameters and $r$ are plotted in Fig. 8 . All the cases are very stable and consistent. The inclusion of $\alpha$ does not produce any shift in the $\Lambda \mathrm{CDM}+r$ parameters. As for the $\Lambda \mathrm{CDM}+\alpha$ model, even in the $\Lambda \mathrm{CDM}+\mathrm{r}+\alpha$ model all the 


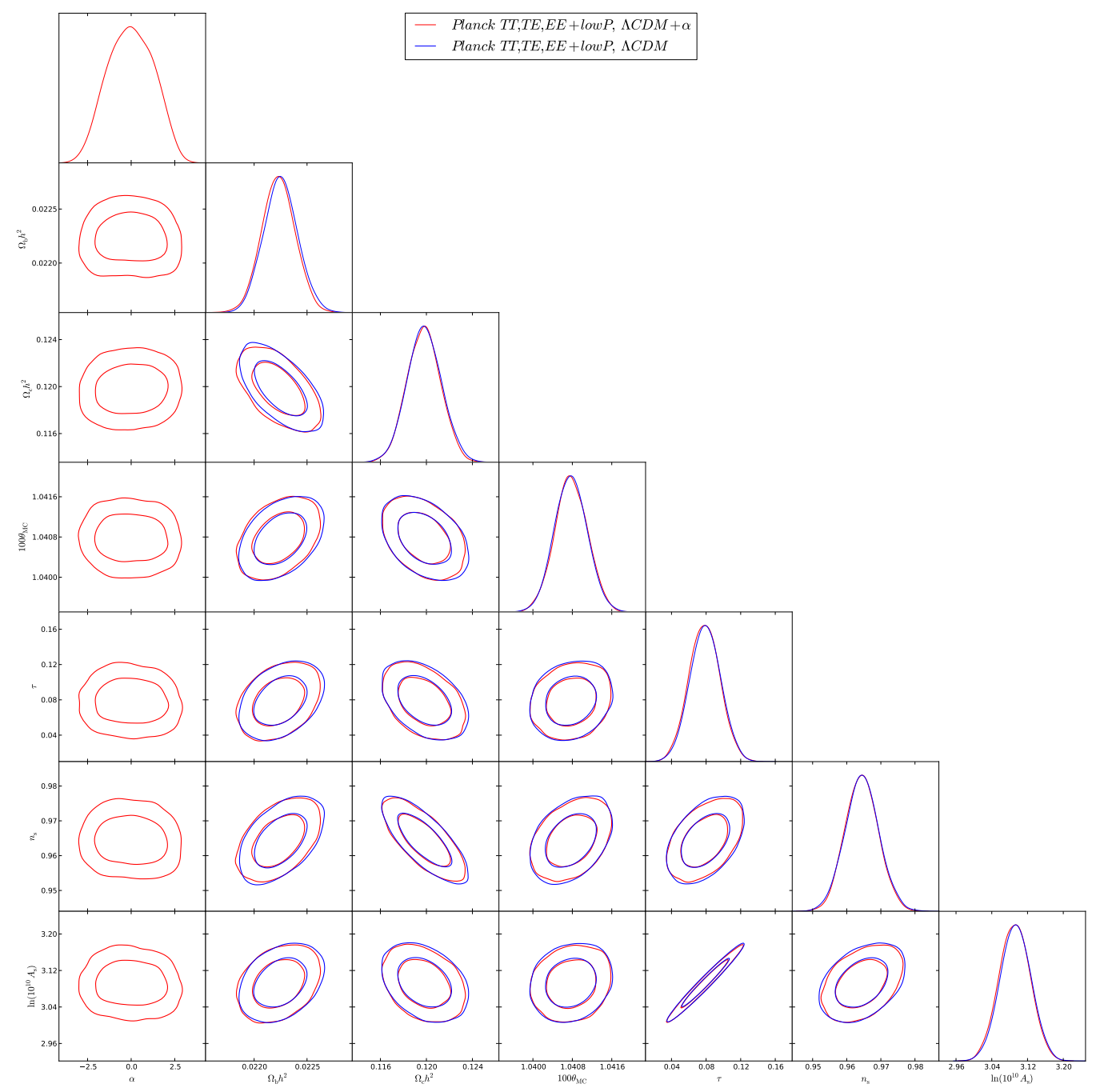

Figure 4: Comparison of the base $\Lambda \mathrm{CDM}$ model parameters and the birefringence angle $\alpha$ from the analysis over the $2 \leq \ell<2500$ range (case B). All the $2 \mathrm{D}$ contour plots are very stable against the inclusion of $\alpha$ in the MCMC sampling. We conclude that $\alpha$ is largely uncorrelated with the other $\Lambda \mathrm{CDM}$ parameters. 
Table 2: Constraints of $\alpha$ for the posterior distributions shown in Fig. 2. Units: deg. $\mathrm{P}$ stands for PlANCK, BKP EE and BKP EE BB for Bicep2/Keck/Planck BB and EE,BB,EB data. unc = unconstrained. $\star$ corresponds to the case in which the six $\Lambda \mathrm{CDM}$ parameters are fixed to the PLANCK best fit.

\begin{tabular}{cccccc}
\hline \hline case & $\alpha_{\text {low }}$ & $\alpha_{\text {high }}$ & $\alpha$ & $\begin{array}{c}\text { model } \\
\Lambda \text { CDM }\end{array}$ & data \\
\hline \hline $\mathrm{A}$ & $5_{-15}^{+12}$ & $0.0 \pm 1.4$ & - & $+\alpha$ & $\mathrm{P}$ \\
$\mathrm{B}$ & - & - & $-0.03 \pm 1.35$ & $+\alpha$ & $\mathrm{P}$ \\
$\mathrm{C}$ & $-0.5_{-12.7}^{+10.2}$ & $0.0 \pm 1.3$ & - & $+\alpha$ & $\mathrm{P}$ \\
$\mathrm{A}$ & $4_{-15}^{+12}$ & $0.0 \pm 1.4$ & - & $+\alpha+\mathrm{r}$ & $\mathrm{P}$ \\
$\mathrm{B}$ & - & - & $0.00 \pm 1.33$ & $+\alpha+\mathrm{r}$ & $\mathrm{P}$ \\
$\mathrm{C}$ & $0.6_{-13.6}^{+9.8}$ & $0.0 \pm 1.3$ & - & $+\alpha+\mathrm{r}$ & $\mathrm{P}$ \\
$\mathrm{A}$ & $4_{-15}^{+12}$ & $0.0 \pm 1.4$ & - & $+\alpha+\mathrm{r}$ & $\mathrm{P}+\mathrm{BKP} \mathrm{BB}$ \\
$\mathrm{B}$ & - & - & $0.02_{-1.41}^{+1.31}$ & $+\alpha+\mathrm{r}$ & $\mathrm{P}+\mathrm{BKP} \mathrm{BB}$ \\
$\mathrm{C}$ & $0.5_{-10.0}^{+9.8}$ & $0.0 \pm 1.4$ & - & $+\alpha+\mathrm{r}$ & $\mathrm{P}+\mathrm{BKP} \mathrm{BB}$ \\
$\mathrm{A}$ & $4_{-15}^{+12}$ & $0.32 \pm 0.27$ & - & $+\alpha+\mathrm{r}$ & $\mathrm{P}+\mathrm{BKP} \mathrm{BB} \mathrm{EE}$ \\
$\mathrm{B}$ & - & - & $0.32 \pm 0.26$ & $+\alpha+\mathrm{r}$ & $\mathrm{P}+\mathrm{BKP} \mathrm{BB} \mathrm{EE}$ \\
$\mathrm{C}$ & $0.9_{-13.6}^{+9.9}$ & $0.32 \pm 0.26$ & - & $+\alpha+\mathrm{r}$ & $\mathrm{P}+\mathrm{BKP} \mathrm{BB} \mathrm{EE}$ \\
$\mathrm{C}^{\star}$ & unc & $0.0 \pm 1.5$ & - & $+\alpha+\mathrm{r}$ & BKP BB \\
$\mathrm{C}^{\star}$ & unc & $0.30 \pm 0.27$ & - & $+\alpha+\mathrm{r}$ & BKP BB EE \\
\hline \hline
\end{tabular}
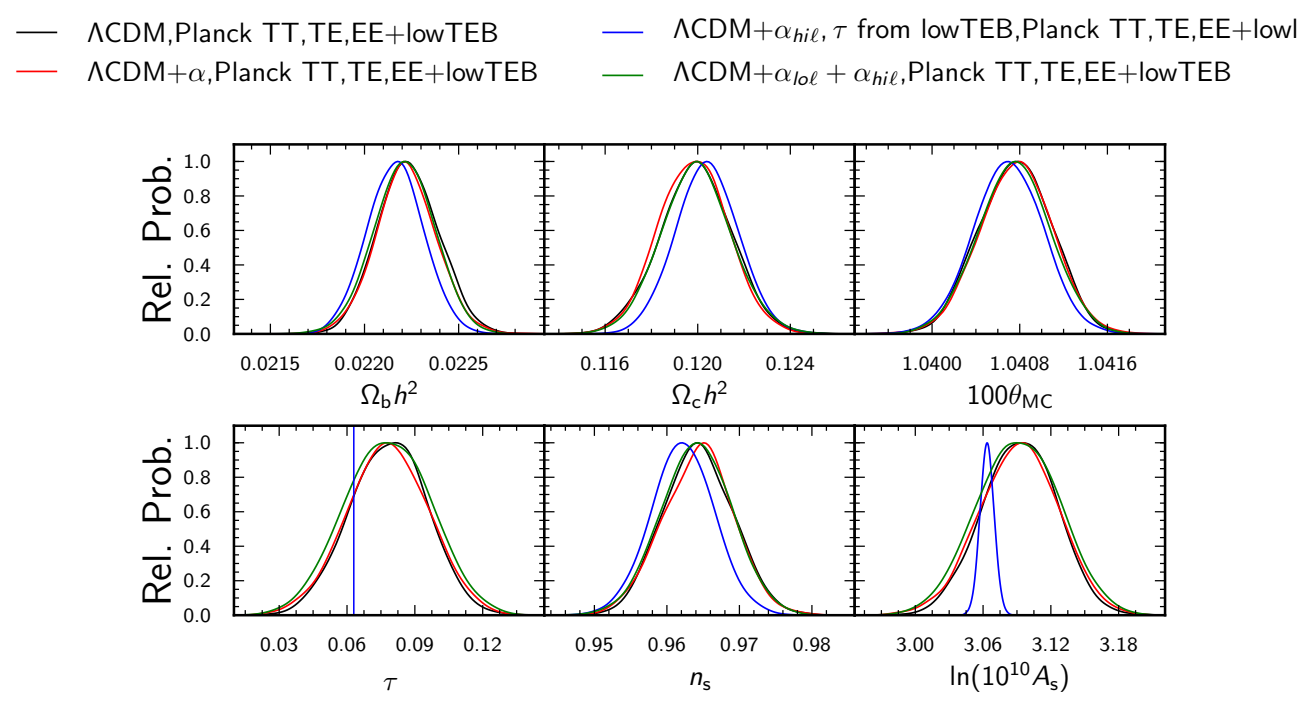

Figure 5: Marginalized posterior distributions for the six main cosmological parameters for the $\Lambda$ CDM when jointly sampled with $\alpha$ for the three considered cases. All the parameters are very compatible among the three cases. 

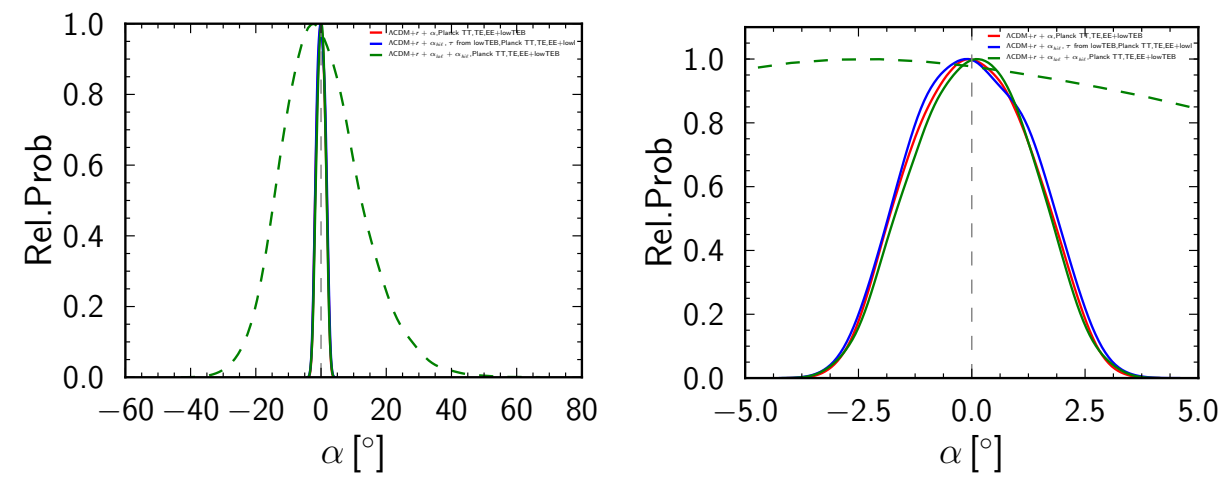

Figure 6: Left panel: Constraints on $\alpha$ from the three considered cases taken into account in $\Lambda \mathrm{CDM}+\mathrm{r}+\alpha$. Blue curves are for case $\mathrm{A}$, red curves for case $\mathrm{B}$ and green curves for case $\mathrm{C}$ (solid green for 30 - 2500 and dashed green for $2-29$ ). Right panel is just a zoom of the right panel. Only PlancK data are employed.
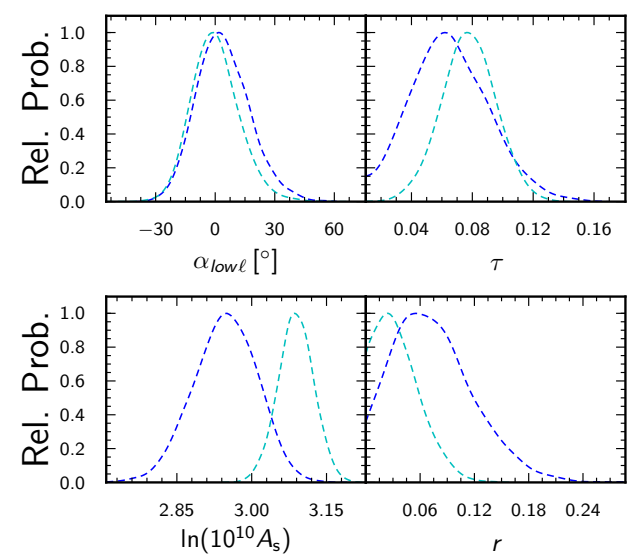

Figure 7: Low $\ell$ run of case A. In $\Lambda \mathrm{CDM}+\mathrm{r}+\alpha$. 

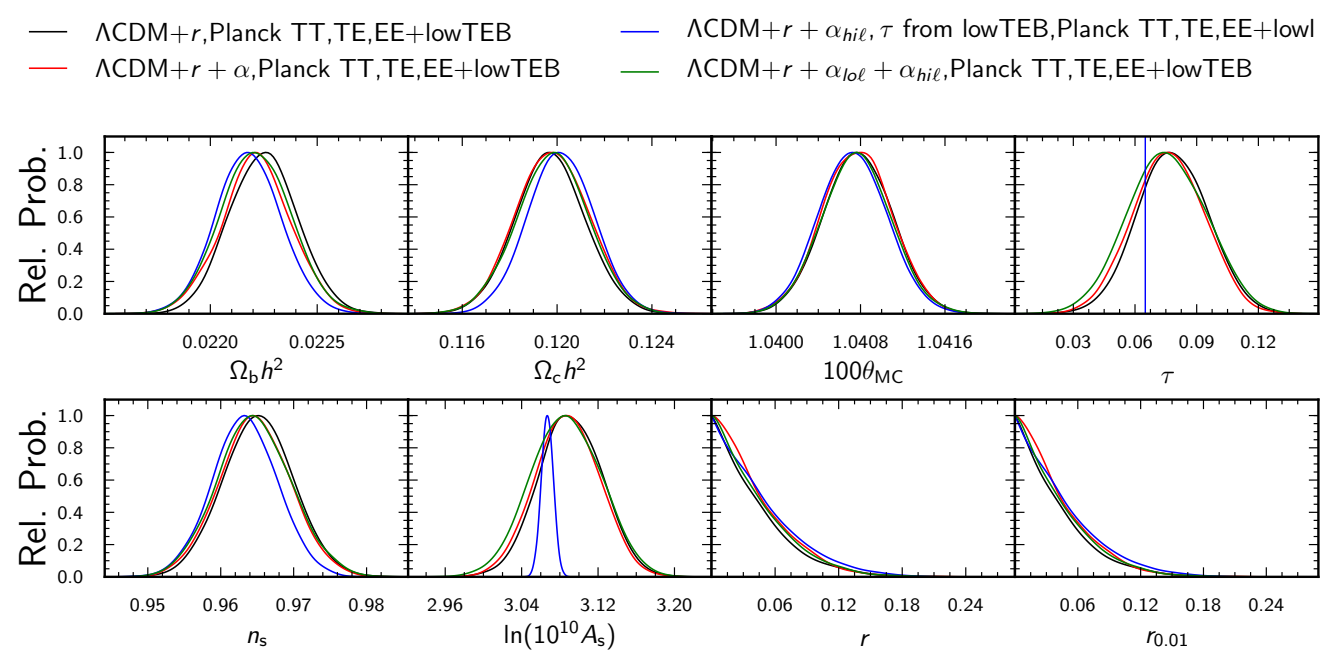

Figure 8: Marginalized posterior distributions for the seven main cosmological parameters for the $\Lambda \mathrm{CDM}+\mathrm{r}$ (blue) and $\Lambda \mathrm{CDM}+r+\alpha$ (red) models from the analysis over the $2 \leq \ell<2500$ range. It is clear that the parameters are very stable against the inclusion of $\alpha$.

PLANCK constraints on $\alpha$ are compatible with zero and numerically very similar.

- PlANCK and BKP data. The probability distribution functions of $\alpha$ are shown in Fig. 9. Blue, red and green solid (dotteddashed) curves are for PLANCK + BKP BB (BKP EE BB) data in the cases $\mathrm{A}, \mathrm{B}$ and $\mathrm{C}$ respectively. Cyan curves are for $\mathrm{BKP}$ data alone (solid for BKP BB and dotted-dashed for BKP EE $\mathrm{BB})$. The corresponding constraints are reported in Table 2. When PLANCK data are employed in combination with BKP BB we notice a change in the shape of the probability distribution function and a bimodal distribution appears. This is due to the fact that the sign of $\alpha_{\text {high }}$ is not constrained when EB and TB spectra are neglected. However the compatibility with zero is at the same level as in the PLANCK data alone analysis. When BKP EE BB data are taken into account in combination with PLANCK, due to the presence of the EB spectrum, the bimodal pattern disappears and a better constraint of $\alpha$ is obtained, i.e. $\alpha=0.32^{\circ} \pm 0.26^{\circ}$ (again, only statistical). In this case the BKP data dominates the combination and the impact of PLANCK data is subleading since what counts more is the EB spectrum. 


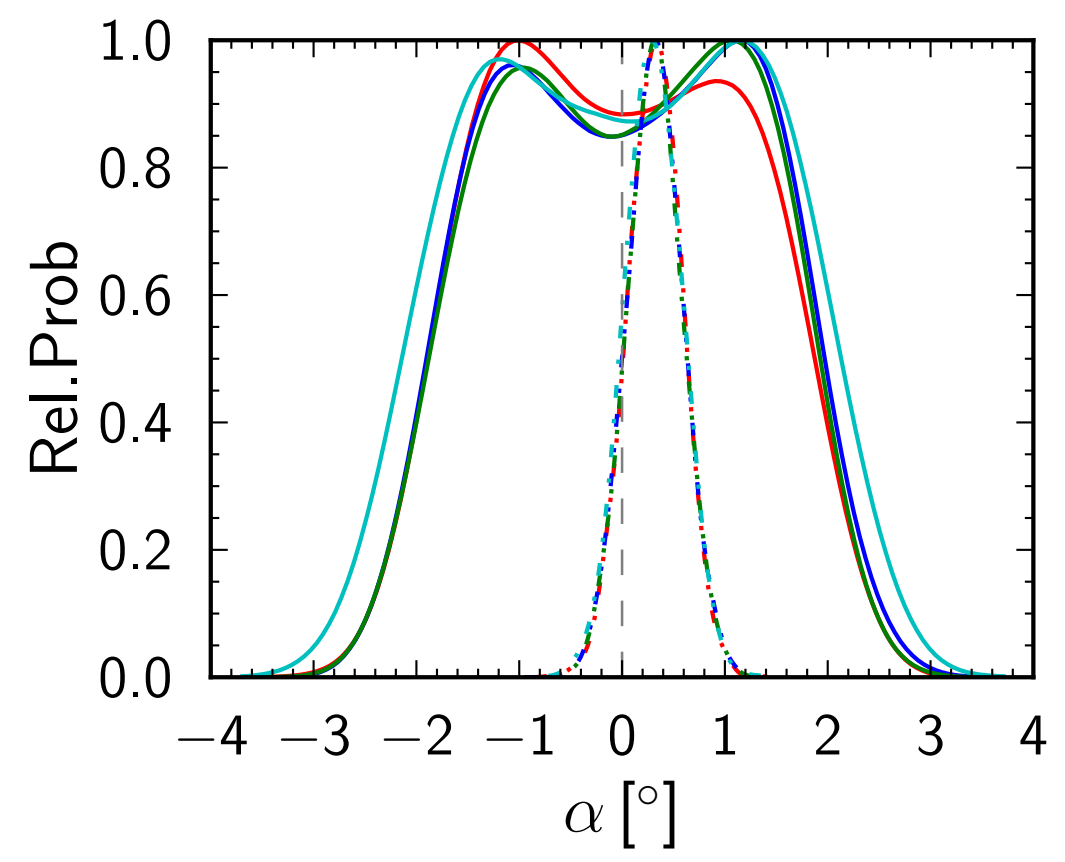

Figure 9: Probability distribution function for $\alpha$. Blue, red and green solid (dotted-dashed) curves are for PLANCK + BKP BB (BKP EE BB) data in the cases $\mathrm{A}, \mathrm{B}$ and $\mathrm{C}$ respectively. Cyan curves are for $\mathrm{BKP}$ data alone (solid for BKP BB and dotted-dashed for BKP EE BB). 


\subsection{Uncertainties from systematic effects}

The most relevant untreated systematic effect that impacts birefringence constraints is a miscalibration of the detectors' optimal polarization angle, i.e. the angle, measured in the telescope reference frame, at which a detector is maximally sensitive to a vertical linear polarization [10]. In the case of PLANCK, ground based measurements cannot be assumed to be representative of in-flight conditions at face value, but rather need to be extrapolated since thermoelastic effects arising in operations can play a role. In-flight measurements are complicated by the scarsity of linearly polarized sources that are bright enough, though the Crab nebula has been attempted as a calibrator [21. For PLANCK, we use the estimate of the error in the polarization orientation evaluated in [23], where it is given at $1^{\circ}$. The original Bicep2 analysis [22] discusses how the TB and EB spectra are rotated to minimize $\mathrm{E}$ to $\mathrm{B}$ mode mixing, by imposing that their residuals from zero are minimized. This process destroys of course any detection power for birefringence below this final rotation angle, which is estimated at $1^{\circ}$ and can thus be taken as a measure of the final systematic budget. In the BKP analysis, the treatment of the Bicep and Keck power spectra is identical to that of Bicep2. We therefore assume a $1^{\circ}$ systematic uncertainty of the instrumental polarization angle for the BKP dataset as well ${ }^{3}$. The same assumption applies to the joint analysis of BKP and PLANCK.

\section{Conclusions}

We have constrained the birefringence angle $\alpha$ employing PLANCK and BKP data either alone or in combination. All our results are well compatible with no detection even without accounting for systematic uncertainty. We find $\alpha=0.0^{\circ} \pm 1.3^{\circ}$ (stat) $\pm 1^{\circ}$ (sys) for PlancK data alone. Similar results are found when we also consider the BB spectrum of BKP data. The inclusion of the EB spectrum in the BKP likelihood makes the constraint on $\alpha$ much tighter, namely $\alpha=0.32^{\circ} \pm$ $0.26^{\circ}$ (stat.) $\pm 1^{\circ}$ (sys.). In the latter case the inclusion of PLANCK data brings little constraining power. This is expected since the current version of the PLANCK Likelihood does not contain TB and EB spectra at high $\ell$. It is possible to forecast that including $\mathrm{BB}, \mathrm{TB}$ and $\mathrm{EB}$ spectra in the high $\ell$ PLANCK likelihood and considering the range of multipoles, i.e. [30-2500], the level of the statistical uncertainty on $\alpha$ can be as low as $\sim 0.03^{\circ}$. This is obtained considering the nominal

\footnotetext{
${ }^{3}$ Notice that for Keck the rotation angle is smaller, i.e. about $0.5^{\circ}$ degrees [24. We assume therefore that the error is dominated by Bicep.
} 
sensitivity of the $143 \mathrm{GHz}$ channel and an exact likelihood approach, see e.g. 25]. The obtained value is compatible with what forecasted in the PLANCK bluebook [26] considering the longer integration time and the improved sensitivity of the used channel. Of course, the quest for reducing the statistical errors would eventually prove meaningless if it is not accompanied by a parallel effort to understand and reduce the systematic error budget affecting polarization rotation constraints.

\section{Acknowledgements}

This paper is based on observations obtained with the satellite PLANCK (http://www.esa.int/Planck), an ESA science mission with instruments and contributions directly funded by ESA Member States, NASA, and Canada. We acknowledge the use of computing facilities at NERSC (USA), of the HEALPix package [23], and of the Planck Legacy Archive (PLA). Research supported by ASI through ASI/INAF Agreement I/072/09/0 for the Planck LFI Activity of Phase E2. MG and LP acknowledges support by the research grant Theoretical Astroparticle Physics number 2012CPPYP7 under the program PRIN 2012 funded by MIUR and by TASP (I.S. INFN).

\section{References}

[1] G. Hinshaw et al. [WMAP Collaboration], Astrophys. J. Suppl. 208, 19 (2013) arXiv:1212.5226 [astro-ph]].

[2] E. Y. S. Wu et al. [QUaD Collaboration], Phys. Rev. Lett. 102, 161302 (2009) arXiv:0811.0618 [astro-ph]].

[3] J. P. Kaufman et al. [BICEP1 Collaboration], Phys. Rev. D 89, 062006 (2014) arXiv:1312.7877 [astro-ph.IM]].

[4] S. M. Carroll, G. B. Field and R. Jackiw, Phys. Rev. D 41, 1231 (1990).

[5] F. Finelli and M. Galaverni, Phys. Rev. D 79, 063002 (2009) arXiv:0802.4210 [astro-ph]].

[6] M. Giovannini, Phys. Rev. D 71, 021301 (2005) hep$\mathrm{ph} / 0410387$.

[7] G. Gubitosi, L. Pagano, G. Amelino-Camelia, A. Melchiorri, and A. Cooray, JCAP 08, 021 (2009).

[8] A. Lue, L. M. Wang and M. Kamionkowski, Phys. Rev. Lett. 83 , 1506 (1999) astro-ph/9812088. 
[9] B. Feng, H. Li, M. z. Li and X. m. Zhang, Phys. Lett. B 620, 27 (2005) hep-ph/0406269.

[10] L. Pagano, P. de Bernardis, G. De Troia, G. Gubitosi, S. Masi, A. Melchiorri, P. Natoli and F. Piacentini et al., Phys. Rev. D 80 (2009) 043522 arXiv:0905.1651 [astro-ph.CO]].

[11] P. A. R. Ade et al. [POLARBEAR Collaboration], Astrophys. J. 794 (2014) 2, 171 arXiv:1403.2369 [astro-ph.CO]].

[12] S. Naess et al. [ACTPol Collaboration], JCAP 1410 (2014) 10, 007 arXiv:1405.5524 [astro-ph.CO]].

[13] S. Dodelson, "Modern cosmology," Amsterdam, Netherlands: Academic Pr. (2003) 440 p

[14] E. Komatsu et al. [WMAP Collaboration], Astrophys. J. Suppl. 180, 330 (2009) arXiv:0803.0547 [astro-ph]].

[15] A. Lewis, Phys. Rev. D 87, no. 10, 103529 (2013) arXiv:1304.4473 [astro-ph.CO]].

[16] A. Lewis and S. Bridle, Phys. Rev. D 66, 103511 (2002) astro$\mathrm{ph} / 0205436$.

[17] N. Aghanim et al. [Planck Collaboration], arXiv:1507.02704 [astro-ph.CO]].

[18] G. Gubitosi, M. Martinelli and L. Pagano, JCAP 1412 (2014) 12, 020 [arXiv:1410.1799 [astro-ph.CO]].

[19] P. A. R. Ade et al. [BICEP2 and Planck Collaborations], Phys. Rev. Lett. 114 (2015) 101301 arXiv:1502.00612 [astro-ph.CO]].

[20] R. Adam et al. [Planck Collaboration], arXiv:1502.05956 [astroph.CO].

[21] R. Adam et al. [Planck Collaboration], arXiv:1502.01587 [astroph.CO].

[22] P. A. R. Ade et al. [BICEP2 Collaboration], Phys. Rev. Lett. 112 (2014) 24, 241101 [arXiv:1403.3985 [astro-ph.CO]].

[23] C. Rosset, M. Tristram, N. Ponthieu, P. Ade, A. Catalano, L. Conversi, F. Couchot and B. P. Crill et al., Astron. Astrophys. 520, A13 (2010) arXiv:1004.2595 [astro-ph.CO]].

[24] P. A. R. Ade et al. [BICEP2 and Keck Array Collaborations], arXiv:1502.00643 [astro-ph.CO].

[25] L. Perotto, J. Lesgourgues, S. Hannestad, H. Tu and Y. Y. Y. Wong, JCAP 0610 (2006) 013 astro-ph/0606227.

[26] J. Tauber et al. [Planck Collaboration], astro-ph/0604069. 